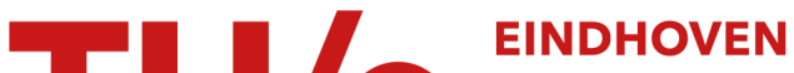 \\ UNIVERSITY OF \\ TECHNOLOGY
}

\section{Decaying quasi-two-dimensional viscous flow on a square domain}

Citation for published version (APA):

van de Konijnenberg, J. A., Flor, J-B., \& Heijst, van, G. J. F. (1998). Decaying quasi-two-dimensional viscous flow on a square domain. Physics of Fluids, 10(3), 595-606. https://doi.org/10.1063/1.869586

DOI:

10.1063/1.869586

Document status and date:

Published: 01/01/1998

\section{Document Version:}

Publisher's PDF, also known as Version of Record (includes final page, issue and volume numbers)

\section{Please check the document version of this publication:}

- A submitted manuscript is the version of the article upon submission and before peer-review. There can be important differences between the submitted version and the official published version of record. People interested in the research are advised to contact the author for the final version of the publication, or visit the $\mathrm{DOI}$ to the publisher's website.

- The final author version and the galley proof are versions of the publication after peer review.

- The final published version features the final layout of the paper including the volume, issue and page numbers.

Link to publication

\section{General rights}

Copyright and moral rights for the publications made accessible in the public portal are retained by the authors and/or other copyright owners and it is a condition of accessing publications that users recognise and abide by the legal requirements associated with these rights.

- Users may download and print one copy of any publication from the public portal for the purpose of private study or research.

- You may not further distribute the material or use it for any profit-making activity or commercial gain

- You may freely distribute the URL identifying the publication in the public portal.

If the publication is distributed under the terms of Article $25 \mathrm{fa}$ of the Dutch Copyright Act, indicated by the "Taverne" license above, please follow below link for the End User Agreement:

www.tue.nl/taverne

Take down policy

If you believe that this document breaches copyright please contact us at:

openaccess@tue.nl

providing details and we will investigate your claim. 


\title{
Decaying quasi-two-dimensional viscous flow on a square domain
}

\author{
J. A. van de Konijnenberg, ${ }^{\text {a) }}$ J. B. Flór, ${ }^{\text {b) }}$ and G. J. F. van Heijst \\ Fluid Dynamics Laboratory, Department of Technical Physics, Eindhoven University of Technology, \\ P.O. Box 513, $5600 \mathrm{MB}$ Eindhoven, The Netherlands
}

(Received 23 June 1997; accepted 4 November 1997)

\begin{abstract}
A comparison is made between experimental, numerical and analytical results for the two-dimensional flow on a square domain. The experiments concern the flow at the interface of a two-layer stratified fluid, evoked by either stirring the fluid with a rake, or by injecting additional fluid at the interface. Two numerical simulations were performed with initial conditions and boundary conditions that correspond approximately with those met in the experiments. The analytical results concern the calculation of the lowest modes of a decaying Stokes flow on a square domain. At late times, the experimental and numerical flows resemble the fundamental mode of the Stokes flow. The damping of small-scale structures by viscous diffusion plays an important role even if the initial Reynolds number is high. In particular, viscous diffusion is likely to be responsible for the appearance of an almost linear relationship between vorticity and stream function in the experiments and the simulations. (c) 1998 American Institute of Physics. [S1070-6631(98)00903-9]
\end{abstract}

\section{INTRODUCTION}

Two-dimensional flows have the property of selforganization, that is, they show a tendency to merge small structures into larger vortices (see, e.g., McWilliams, ${ }^{1}$ Legras, Santangelo and Benzi, ${ }^{2}$ Boubnov, Dalziel and Linden ${ }^{3}$ ). On a finite domain, the growth of these vortices is limited by the boundaries. The flow then often results in an organized state, consisting of a small number of vortex cells that fill the entire domain. The appearance of such an organized state is rather insensitive to the way the flow is produced. Both a continuously driven flow and a freely evolving flow tend to organize themselves into large cells, even if the forcing or the initial flow field do not suggest the formation of particular large-scale structures. Although a general theory for the organized state appears to be difficult to derive, it is recognized that self-organization is a property of two-dimensional flows with very small, or even zero viscosity.

Montgomery and Joyce ${ }^{4}$ and Robert and Sommeria ${ }^{5}$ proposed an approach based on statistical methods to determine a functional relationship between vorticity $\omega$ and stream function $\psi$ of the organized state of a freely evolving twodimensional flow. Their methods are technically different, but both rely on the counting of allowable dynamical states. The final equilibrium state of an initial vorticity distribution is calculated by introducing and maximizing the entropy of the vorticity field. Since these theories rely on the conservation of certain integral quantities such as the energy of the flow field, they apply only to strictly inviscid flows. In the method by Montgomery and Joyce, point vortices are introduced to represent the vorticity field. A well-known result of this approach is that if the vorticity distribution consists of

\footnotetext{
${ }^{a}$ Present address: Ris $\varnothing$ National Laboratory, Optics and Fluid Dynamics Dept., P.O. Box 49, 4000 Roskilde, Denmark.

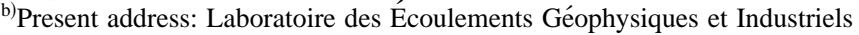
(UJF-CNRS-INPG), B.P. 53X-38041 Grenoble Cedex 09, France.
}

the same number of positive and negative point vortices with equal strength, the relation between vorticity and stream function becomes a hyperbolic sine:

$$
\omega=\omega_{0} \sinh (\beta \psi) .
$$

The method by Robert and Sommeria is more complex and departs from a regular vorticity distribution. If the initial flow field consists of thin shear layers in an otherwise irrotational environment, such that the distribution of positive and negative vorticity is symmetric, the hyperbolic sine result of Montgomery and Joyce is recovered. More general conclusions about the final vorticity distribution are found as well, in particular that $\omega$ is a strictly increasing function of $\psi$. It is the assumption of inviscid flow as well as the technical difficulties to construct solutions for more general vorticity distributions that has hampered a widespread use of this method as yet. In addition to the two mentioned articles, several contributions were made by Salmon, Holloway and Hendershott, ${ }^{6}$ Robert and Sommeria ${ }^{7}$ and Montgomery, Shan and Matthaeus. ${ }^{8}$

Less recognized is the fact that the emergence of a largescale structure may also be the result of viscous diffusion in the relaxing flow. In analogy with heat conduction in a plate, small inhomogeneities disappear more quickly than larger ones, so that eventually a fundamental mode remains. Even when the Reynolds number is still large, viscous diffusion may enhance the formation of an organized state, and have considerable influence of the structure of the vortex cells.

In this paper, we present experimental, numerical and analytical results for two-dimensional flow on a square domain. We consider flows that are driven for a finite time; the emphasis is put on the evolution after the forcing is stopped. It will be seen that even for very different types of initial forcing, both the experiments and the numerical simulations lead to a state that corresponds to the lowest mode of a decaying Stokes flow. 
The experiments were performed in a square tank, filled with a two-layer stratified fluid. Two initial conditions were used: $(i)$ a flow field created by a temporary injection of additional fluid by two opposite jets, and (ii) an initially disorganized motion of small vortices, generated by moving a row of small bars through the fluid (see also Flor ${ }^{9}$ ). As a consequence of the stratification, the flow soon becomes planar, and at the midplane it is then governed to leading order by (see Flór and Van Heijst ${ }^{10}$ )

$$
\frac{\partial \omega}{\partial t}+u \frac{\partial \omega}{\partial x}+v \frac{\partial \omega}{\partial y}=\nu \nabla_{\mathrm{h}}^{2} \omega+\nu \frac{\partial^{2} \omega}{\partial z^{2}}
$$

with $\omega$ the vertical component of the vorticity, $u$ and $v$ the Cartesian components of the horizontal velocity, and $\nu=0.01$ $\mathrm{cm}^{2} / \mathrm{s}$ the kinematic viscosity of water at room temperature.

The numerical simulations consist of the integration of the incompressible two-dimensional vorticity equation, that is, equation (2) without the term $\nu \partial^{2} \omega / \partial z^{2}$. The initial conditions were designed to correspond with the situation in the experiments.

The analytical results consist of an extension of the earlier work by Taylor ${ }^{11}$ on the buckling of a clamped plate under a distributed load, a problem that appears to be connected with the diffusion of vorticity on a domain with noslip boundary conditions. With the aid of the computer, Taylor's implicit results have been evaluated and applied to the Stokes flow in a rectangle (see also Van de Konijnenberg ${ }^{12}$ ).

The two-dimensional nature of the flow under consideration allows the introduction of a stream function $\psi$ according to

$$
u=\frac{\partial \psi}{\partial y} ; \quad v=-\frac{\partial \psi}{\partial x} .
$$

An important diagnostic tool in the study of two-dimensional vortex structures is the graph of $\omega$ versus $\psi$, the so-called scatterplot. Scatterplots derive their main importance from the property of stationary inviscid two-dimensional flows that the advective term in the vorticity equation vanishes, so that for each vortex, there is a functional relationship between $\omega$ and $\psi$. However, it will be shown in this paper that also in the case of an equilibrium between $\partial \omega / \partial t$ and $\nu \nabla^{2} \omega$, there can be a strong correlation between $\omega$ and $\psi$.

The further organization of this paper is as follows. The experimental set-up is described in section II, followed by a brief discussion of the numerical method in section III. The experimental and numerical results are discussed in section IV. In section $\mathrm{V}$, the theory for a decaying viscous flow is presented. Finally, some general conclusions are drawn in section VI.

\section{EXPERIMENTAL SET-UP}

The experiments were performed in a square tank with side $L=80 \mathrm{~cm}$, filled with a two-layer stratified fluid. This configuration consisted of a lower layer of salty water (density $1.10 \mathrm{~g} / \mathrm{cm}^{3}$ ) and an upper layer of fresh water (density $1.00 \mathrm{~g} / \mathrm{cm}^{3}$ ). Both layers had a depth of $10 \mathrm{~cm}$. The stratification was made by slowly releasing the salty water under the fresh water through a diffuser.
The first type of initial flow was generated by stirring the fluid with a rake consisting of 17 aligned bars $14 \mathrm{~mm}$ apart, each with diameter $d=6 \mathrm{~mm}$. The velocity of the stirring motion was typically $50 \mathrm{~cm} / \mathrm{s}$, yielding a turbulent flow with a Reynolds number $U d / \nu$ of the order of 3000 . The rake was drawn through the fluid over the full depth of the tank, but dye visualizations indicate that the turbulence outside the interfacial region disappears quickly, so that only a planar flow in the interfacial layer remains. Judging from the distribution of a large number of neutrally buoyant particles, the thickness of the interface after the turbulence has settled is about $3 \mathrm{~cm}$.

The second type of initial flow was produced by two horizontal jets, generated by nozzles with diameter $d=4 \mathrm{~mm}$, symmetrically placed at two opposite tank walls. The injection nozzles were located at the level of the interface between the two layers, and were pointed along the sidewall in opposite directions. The injected fluid had an intermediate density, so that it remained trapped at the interface. The injection fluid simply flowed under gravity from a second vessel into the experimental tank. Since this vessel had a relatively large cross section, the flow rate of the injection could be kept approximately constant. The fluid was injected for a period of about $10 \mathrm{~min}$. Immediately after the injection was stopped, the nozzles were gently removed; this time is referred to as $t=0$. The flow rate $Q$ for each jet was $4 \mathrm{ml} / \mathrm{s}$, so that the injection Reynolds number, defined as $Q /(\pi d \nu)$, had a value of about 300 .

The flows were visualized with polystyrene particles with density $1.07 \mathrm{~g} / \mathrm{cm}^{3}$, floating at the interface between the two layers, and with dye (sodium fluorescein and rhodamine G). In order to obtain quantitative information about the velocity field, streak photographs of the moving particles were taken with a camera suspended above the tank. By digitizing the streaks, the velocity of each particle could be obtained. These data were supplemented with a large number of zero vectors at the sidewalls, and interpolated to a rectangular grid of $30 \times 30$ grid points by using cubic splines. From the coefficients of the spline expansion, the vorticity field could be derived. Subsequently, the stream function was determined by solving numerically the Poisson equation

$$
\nabla^{2} \psi=-\omega
$$

with boundary condition $\psi=0$. More detailed information about this method of data processing can be found in Nguyen Duc and Sommeria ${ }^{13}$ and Flór and Van Heijst. ${ }^{10}$

\section{NUMERICAL METHOD}

The numerical simulations were based on an ADI (Alternating Direction Implicit) method for solving the twodimensional vorticity equation

$$
\frac{\partial \omega}{\partial t}+u \frac{\partial \omega}{\partial x}+v \frac{\partial \omega}{\partial y}=\frac{1}{R e} \nabla^{2} \omega
$$

with the condition of zero velocity at the boundaries. A detailed discussion of this scheme is given by Fletcher. ${ }^{14}$ The vorticity equation has been non-dimensionalized with a typical velocity $U$ and the side $L$ of the domain, so the Reynolds 
a)
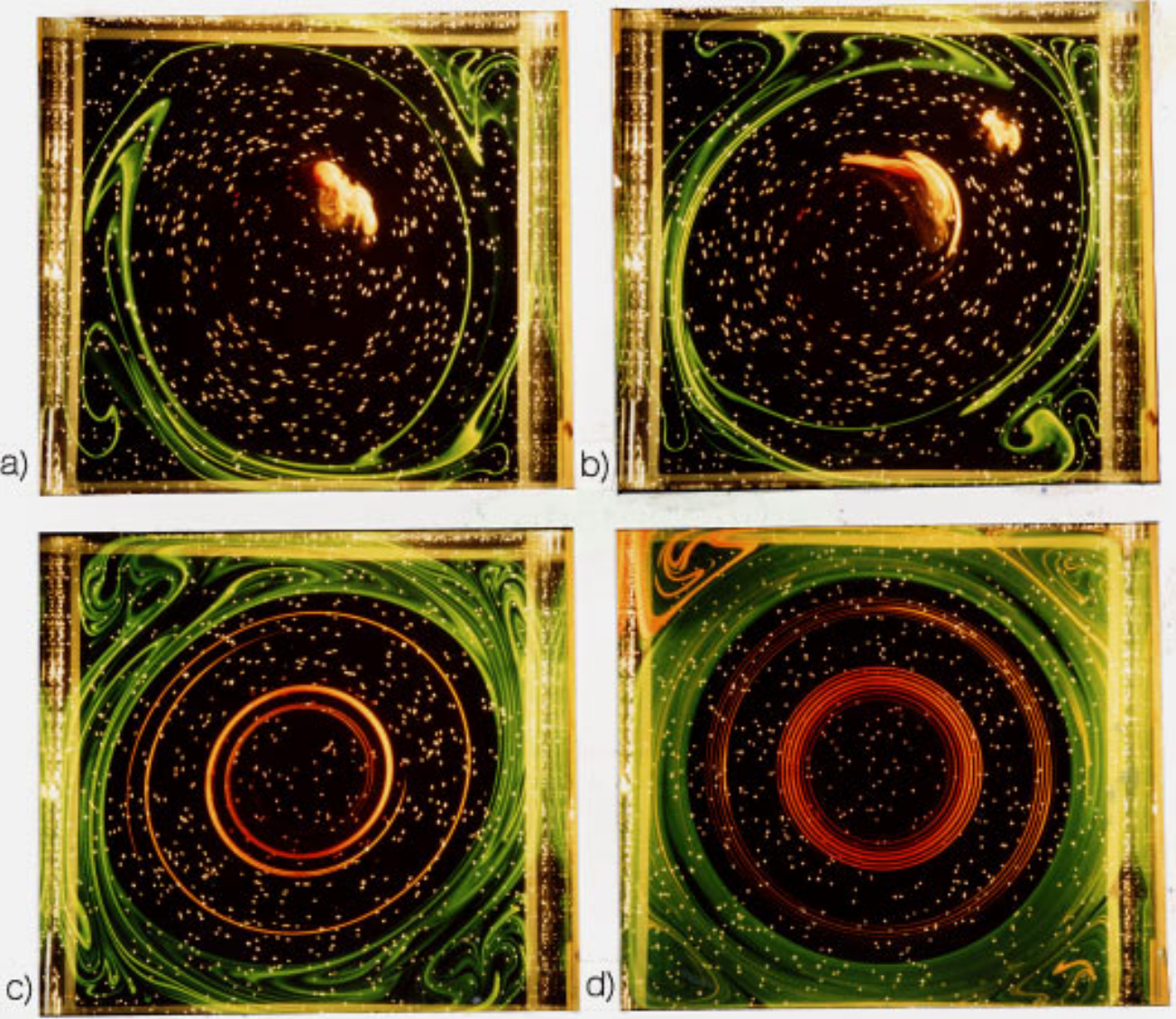

FIG. 1. Dye visualization of the rake-forced flow. The green dye was added at $t=30 \mathrm{~min}$; the red dye was added at $t=40 \mathrm{~min}$ and at $t=42 \mathrm{~min}$. The pictures were taken at (a) $41 \mathrm{~min}$; (b) $43 \mathrm{~min}$; (c) $70 \mathrm{~min}$ and (d) $154 \mathrm{~min}$

number is defined here as $U L / \nu$. The flow is solved on a square grid of $100 \times 100$ points with equidistant spacings $\Delta x$ and $\Delta y$. The numerical scheme is accurate to second order in $\Delta x$ and $\Delta y$ and in the time step $\Delta t$. A variable time step was used, small when the flow changed rapidly, and large in the end of the experiment when the flow changed only slowly.

Unfortunately, the Reynolds number could not be chosen higher than about 500, since this would require a finer grid and smaller time steps, and therefore too much computer time. However, it is the purpose of the present paper to demonstrate some general properties of the flow on a square domain, and it will appear that both the experimental and the numerical results lead to the same conclusion with respect to the structure of the velocity field after a long time.

The numerical method was used to simulate the flow generated by stirring with a rake. The initial flow field was modelled with an array of vortices given by

$$
\begin{aligned}
\omega(x, y)= & A \cos (8 \pi x) \cos (8 \pi y) \\
& \times \exp \left\{-\alpha\left[\left(x-x_{\mathrm{c}}\right)^{2}+\left(y-y_{\mathrm{c}}\right)^{2}\right]\right\}
\end{aligned}
$$

with both $x$ and $y$ running from 0 to 1 , and $A$ the amplitude (see Fig. 4(a)). This initial condition consists of 8 vortices in each coordinate direction, and has a Gaussian vorticity amplitude envelope. It was verified that when the initial condi- tion is symmetric with respect to inversion of $x$ and $y$, the flow preserved its symmetry. In the following computations, this symmetry was broken by the eccentric position $\left(x_{\mathrm{c}}, y_{\mathrm{c}}\right)$ of the maximum of the amplitude envelope. Although the initial flow field is organized, it has the main features of the experimental flow field: a large number of interacting vortices that are asymmetric with respect to the boundaries. Simulations were carried out with $R e=400, A=5$ and $\alpha=0.5$. The position of the envelope maximum was only slightly off-centred $\left(x_{\mathrm{c}}=0.56\right.$ and $\left.y_{\mathrm{c}}=0.58\right)$.

\section{EXPERIMENTAL AND NUMERICAL RESULTS}

\section{A. Rake-forced flow}

A dye visualization of the rake-forced flow is displayed in Fig. 1. It took about $15 \mathrm{~min}$ before a coherent central vortex was formed. The fluorescein dye (green) was added at $t=30 \mathrm{~min}$ at the middle of the "upper" wall. The rhodamin $\mathrm{G}$ (red) was added at $t=40 \mathrm{~min}$, just before the picture shown in Fig. 1(a) was taken. The green dye shows that the central vortex is elliptical, and performs a clockwise rotation (see Figs. 1(a) and (b)). In the initial stage (Figs. 1(a) and (b)), the angular velocities of the green and the red dye were approximately 0.012 and $0.046 \mathrm{rad} / \mathrm{s}$, respectively, indicating 

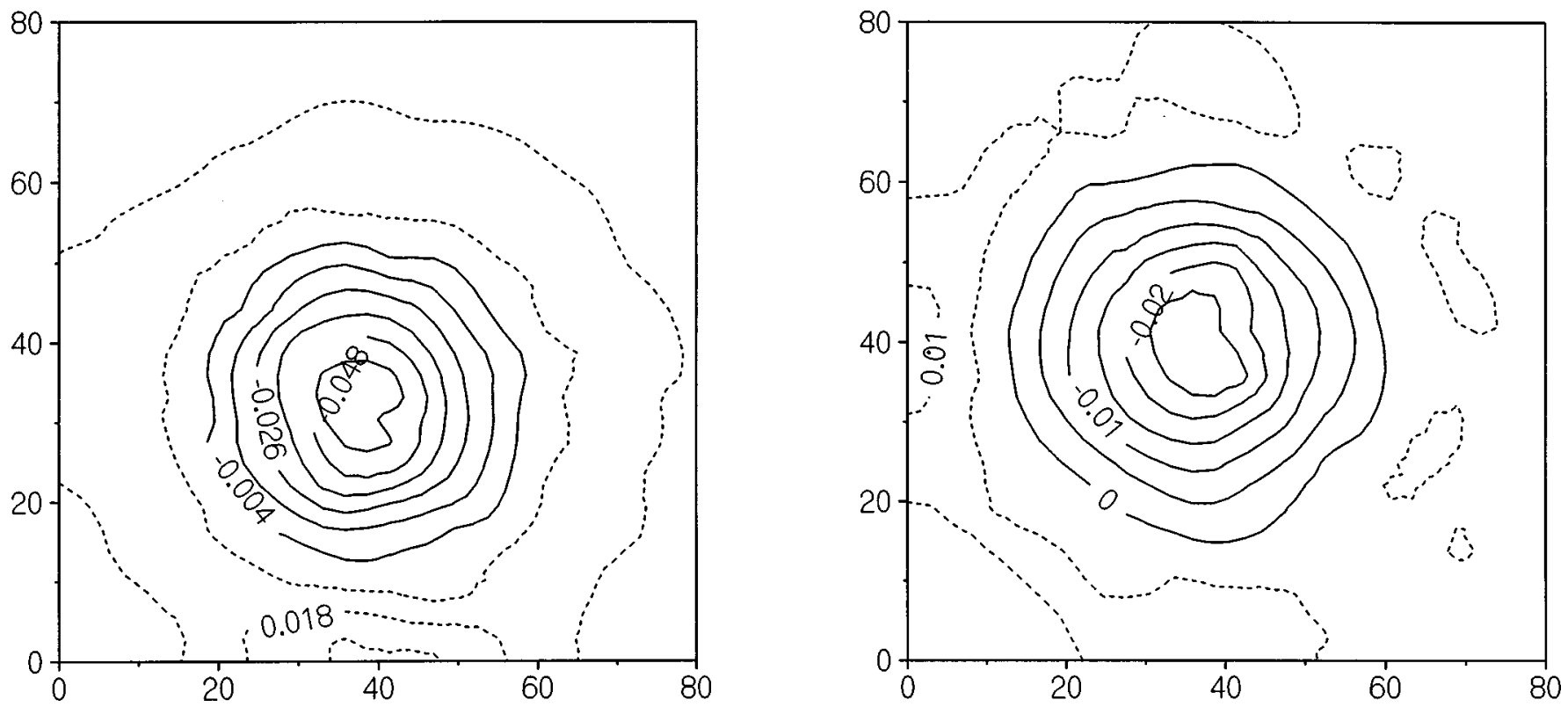

FIG. 2. Vorticity-contour plots of the rake-forced flow at (a) $35 \mathrm{~min}$ and (b) $95 \mathrm{~min}$ after the forcing was stopped.

a differential rotation of the central vortex. The vorticity corresponding to the shear layer at the sidewalls evolves into two small vortices on the long sides of the elliptical core vortex. In the course of the experiment, the shape of the central vortex becomes circular, as can be seen in Fig. 1(d).

Close inspection of Fig. 1(d) reveals another feature of the flow evolution: The red dye in the central part of the vortex is distributed along a spiral with sharp edges. This indicates that no mixing occurs in the core region of the vortex, and the dye patch is only stretched due to the differential angular velocity. In contrast, the green dye, released much closer to the wall, is eventually distributed more or less homogeneously in a band around the vortex. This is indicative of mixing that has occurred in this area, owing to the unsteadiness of the flow near the sides and corners of the tank (see Figs. 1(a), (b) and (c)). At later times (see Fig. 1(d)) the flow becomes more steady, consisting of a circular central vortex and four corner cells. Orange dye (tectilon rot), released in some of the corner cells just before the picture of Fig. 1(d) was taken, is observed to remain trapped in these cells, indicating poor mixing at this stage.

Figure 2 shows experimental vorticity contours at $t=35$ $\min$ and $t=95 \mathrm{~min}$. At $t=35 \mathrm{~min}$, the central vortex has an off-centred position, and a closer inspection of the experimental data reveals that the center of this vortex performs an oscillatory motion. The excursions gradually become smaller, until finally the core vortex settles at a central position in the tank. Although the global distribution of the vorticity contours is correct, it is possible that small-scale features, such as the irregularity of the contours close to the center, are due to experimental error. In particular, the low velocities at the center are difficult to measure accurately. In view of the virtually circular appearance of the dye lines at late times, we believe that the central vorticity contours at late times should be circular as well.

Figure 3 shows the $\omega, \psi$-scatterplots of the rake-forced flow. The peak in the each graph corresponds to the core of the vortex. The negative vorticity represents the flow near the boundaries; in the corners the vorticity is close to zero. The shape of the $\omega, \psi$-relation is similar to that obtained for a stationary isolated monopolar vortex in an unbounded flow as shown by Flór and Van Heijst. ${ }^{15}$ All scatterplots show an upward curvature, but the departures from a linear profile are small and slowly decrease in the course of the experiment.

The numerical results for the multi-vortex initial condition are given in Fig. 4. The initial condition is shown in Fig. 4(a). Figure 4(b) shows that at $t=20 \mathrm{~min}$, the number of vortices has already been reduced. In later stages, only a central vortex surrounded by a ring of positive vorticity remains. Initially, this central vortex is elliptical, but as in the experiment (Fig. 1), it becomes approximately circular later on (see Fig. 4(f)).

Throughout the flow evolution, one observes bands of large-amplitude vorticity at the boundaries, which represent the viscously produced wall vorticity associated with the noslip boundary condition. In fact, it is doubtful whether the finite-difference method used here captures these small-scale effects completely. Although the flow in the early stages is certainly affected by these intense vorticity filaments (which are continuously advected away from the walls, into the interior of the domain), eventually the flow takes on the appearance as in Fig. 4(d), i.e. one large central cell with an oppositely-signed vorticity shield at the walls. This has been confirmed by recent high-resolution simulations based on a spectral method (Clercx, Maassen, and Van Heijst ${ }^{16}$ ).

Figure 5 displays the $\omega, \psi$-scatterplots according to the numerical simulations. Initially, the $\omega, \psi$-relation shows a vorticity peak at the $\psi=0$ axis, which corresponds with the viscously generated vorticity at the boundaries (see Fig. 5(a)). The width of these bands of oppositely signed wall vorticity increases in the course of the experiment, and the extremal values of the vorticity become smaller. The scatter 

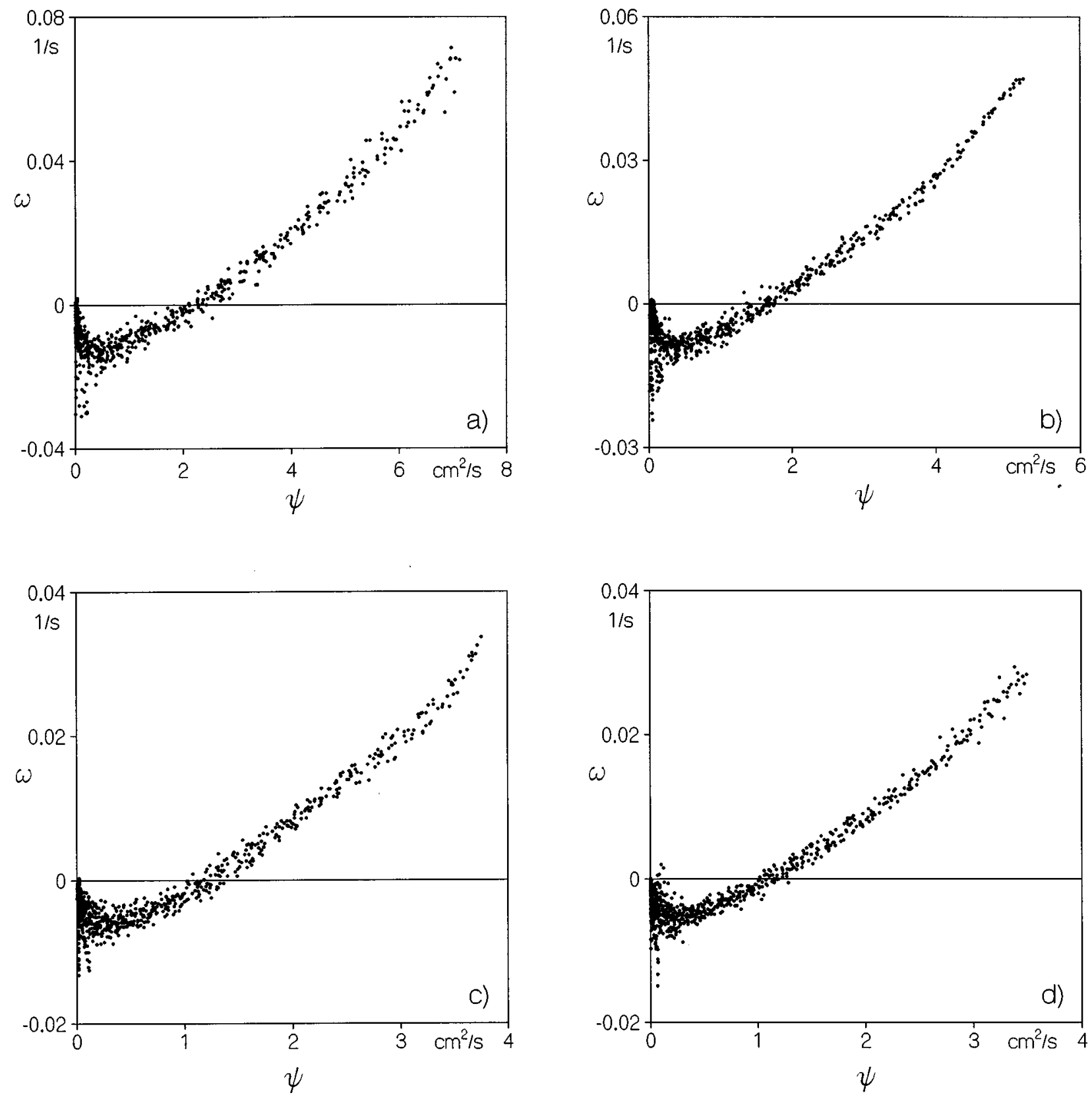

FIG. 3. Scatterplots of the rake-forced flow at (a) $35 \mathrm{~min}$; (b) $55 \mathrm{~min}$; (c) $85 \mathrm{~min}$ and (d) $105 \mathrm{~min}$.

in the $\omega, \psi$-graphs is seen to disappear gradually, and eventually a well-defined, almost linear profile remains.

\section{B. Jet-forced flow}

A sequence of experimentally determined vorticity contour plots and $\omega, \psi$-scatterplots for the jet-forced flow is shown in Figs. 6 and 7, respectively. Soon after the start of the injection, a quasi-steady large-scale flow was established. This flow is characterized by a more or less uniform vorticity in the central part of the domain, a feature that is still easily observed during some time after the forcing was stopped, see Figs. 6(a) and 7(a). The set-up with two jets bears resemblance with a configuration used by Boubnov et al., ${ }^{3}$ who used a steady forcing with eight sources and eight sinks at the perimeter of a square tank filled with a stratified fluid, and also found a quasi-steady flow with a uniform-vorticity core.

Figures 6(b), (c) and (d) show the vorticity distribution at later stages in the flow evolution. It appears that the flow remains more symmetric than in the case of the rake-forced flow. In the course of the experiment, the horizontal part of the $\omega, \psi$-graph (Fig. 7) is seen to evolve into a linear branch with nonzero slope, similar to what is observed in the evolving rake-forced flow (see Fig. 3(d)). Thus, although the two forcings lead to a different evolution of the flow, in both cases the relationship between $\omega$ and $\psi$ eventually becomes approximately linear.

Numerical simulations were also carried out for this case, with the jets modelled by two strips of double-signed 


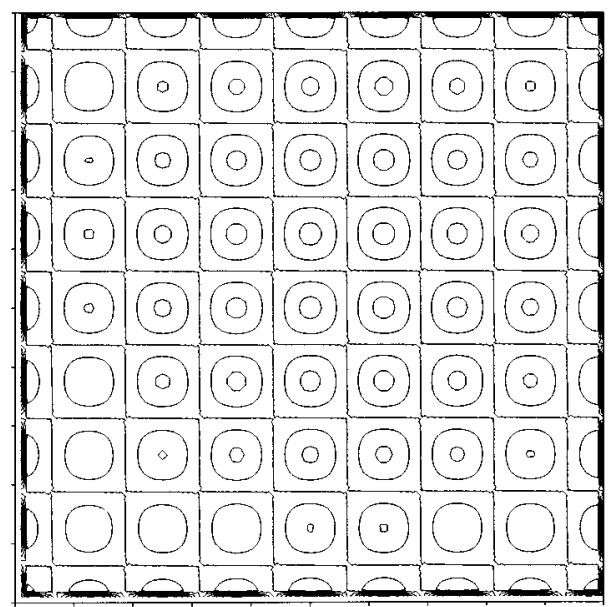

(a)

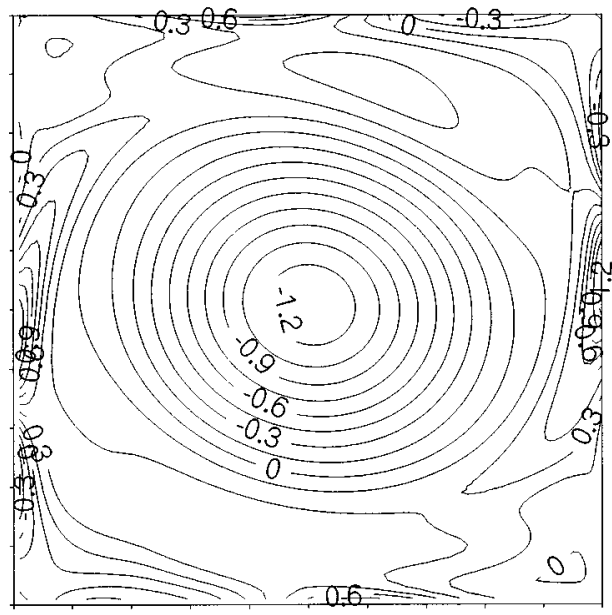

(c)

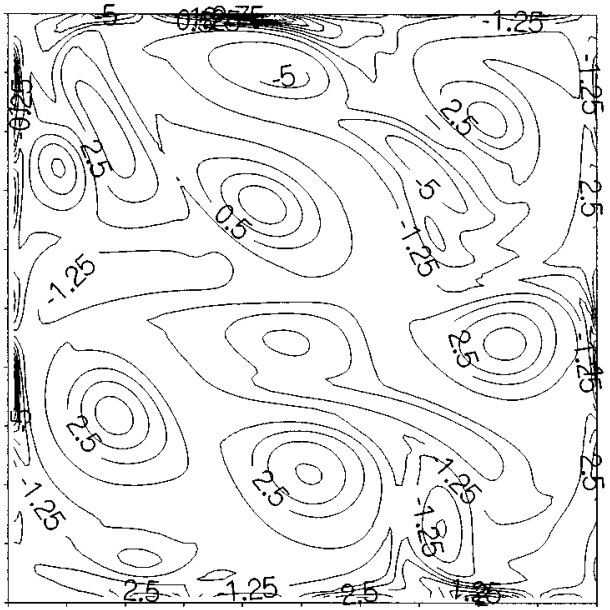

(b)

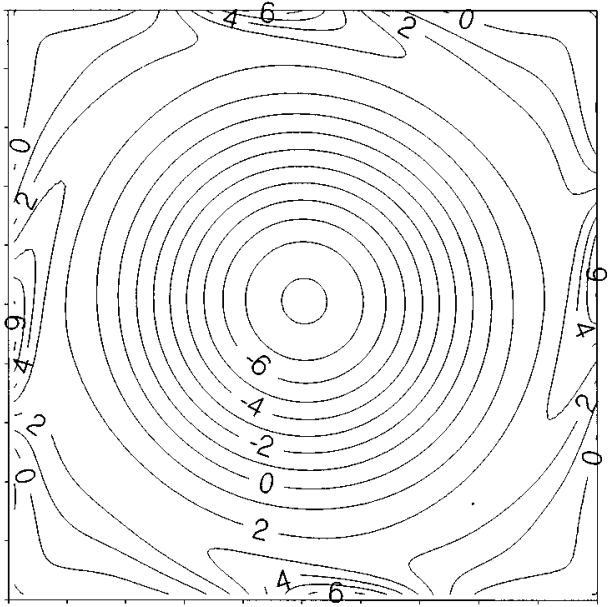

(d)

FIG. 4. Numerical results for the rake-forced flow. The graphs show the vorticity contours at (a) 1 min; (b) 20 min; (c) 145 min and (d) 270 min. The distances between the contour levels are (a) 0.088 ; (b) 0.00375 ; (c) 0.0015 and (d) 0.001 . Numerical parameters: $\operatorname{Re}=400, A=5, \alpha=0.5$.

large-amplitude vorticity in an otherwise vorticity-free domain at $t=0$. Although this approach is rather crude, the details of the initial flow structure were found not to affect the flow in the ultimate stage, which was indistinguishable from that shown in Figs. 4(d) and 5(d): the large cell is again characterized by a linear $\omega, \psi$-relationship. A similar tendency to a linear $\omega, \psi$-relationship was observed by Boubnov et $a .^{3}$ after the forcing was stopped.

\section{THEORY}

In this section, we focus on the decay of a twodimensional flow on a square domain given by $-a<x<a$ and $-a<y<a$ in the limit $R e=0$. This limiting case implies the absence of the advective term in the vorticity equation. Thus, the aim is to find solutions of the vorticity equation

$$
\frac{\partial \omega}{\partial t}=\nu \nabla^{2} \omega
$$

with zero-velocity boundary conditions. The time dependence in this problem can be eliminated by separation in $x$ and $y$ on one hand, and $t$ on the other hand. This leads to exponentially decaying modes, which may be superimposed to yield the full time-dependent solution for any given initial flow field:

$$
\omega(x, y, t)=\sum_{\mu} C_{\mu} \omega_{\mu}(x, y) e^{-\mu \pi^{2} \nu t / a^{2}} .
$$

The constants $\pi, \nu$ and $a$ in the exponent result in dimensionless eigenvalues $\mu$, and prove to be convenient in the following calculation. For each value of $\mu$, we find a Helmholtz equation for $\omega(x, y)$ :

$$
\nabla^{2} \omega+\mu \frac{\pi^{2}}{a^{2}} \omega=0
$$

with both $\partial \psi / \partial x$ and $\partial \psi / \partial y$ being zero at the walls. Since 

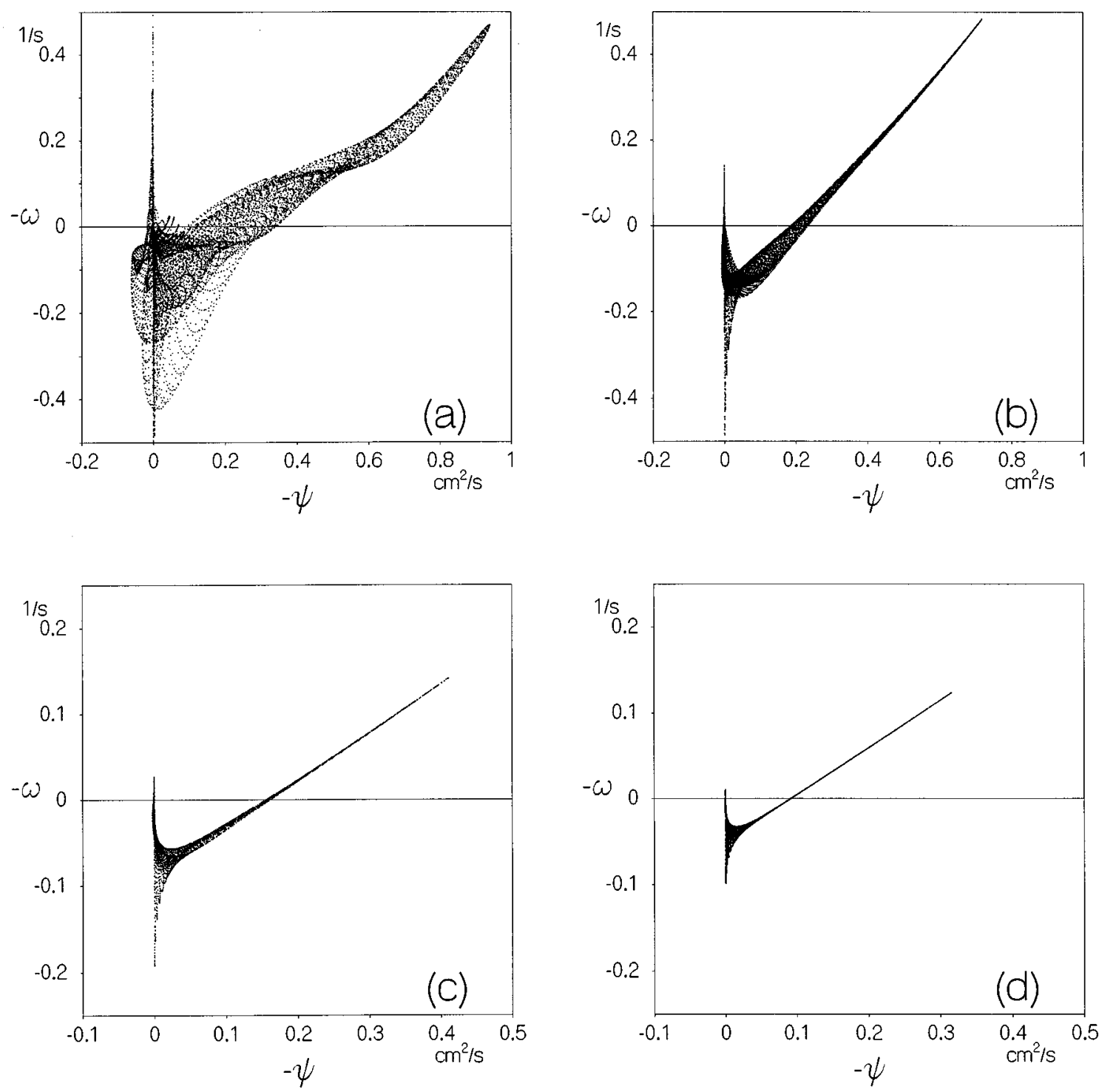

FIG. 5. Scatterplots of $\omega$ versus $\psi$ of the rake-forced flow according to the numerical simulation at (a) $85 \mathrm{~min}$; (b) $125 \mathrm{~min}$; (c) $187 \mathrm{~min}$ and (d) $270 \mathrm{~min}$.

the boundary conditions concern the stream function instead of the vorticity, (9) should be regarded as a fourth-order problem in the stream function $\psi$ :

$$
\nabla^{4} \psi+\mu \frac{\pi^{2}}{a^{2}} \nabla^{2} \psi=0 .
$$

The boundary conditions imply that $\psi$ has the same value along the four walls; this value is taken to be zero.

This mathematical problem has been studied earlier by Taylor $^{11}$ in the context of the displacement of a clamped rectangular plate subjected to a buckling load when all edges are clamped. The problem also bears resemblance with the inhomogeneous biharmonic equation

$$
\nabla^{4} \psi=q(x, y) .
$$

with similar boundary conditions, occurring in the theory of bending of clamped plates under a distributed load (Timoshenko and Woinowsky-Krieger, ${ }^{17}$ Meleshko and Gomilko ${ }^{18}$ ).

Since the lowest mode in a square tank is expected to be symmetric with respect to both $x=0$ and $y=0$, only even functions of $x$ and $y$ are considered in this analysis. Calculation of modes consisting of odd functions is unlikely to introduce fundamentally new problems. The extension of this problem to a rectangular geometry is straightforward too, but gives rise to somewhat more cumbersome formulae.

The problem is solved by writing the stream function $\psi$ as a combination of two series:

$$
\psi(x, y)=\psi_{1}(x, y)+\psi_{2}(x, y)
$$

with

$$
\begin{aligned}
\psi_{1}= & \sum_{m=0}^{\infty} A_{m} \frac{\cos \left(m+\frac{1}{2}\right) \pi x / a}{(-1)^{m}\left(m+\frac{1}{2}\right)} \\
& \times\left(\frac{\cosh \sqrt{\left(m+\frac{1}{2}\right)^{2}-\mu} \pi y / a}{\cosh \sqrt{\left(m+\frac{1}{2}\right)^{2}-\mu} \pi}-\frac{\cosh \left(m+\frac{1}{2}\right) \pi y / a}{\cosh \left(m+\frac{1}{2}\right) \pi}\right)
\end{aligned}
$$

and 


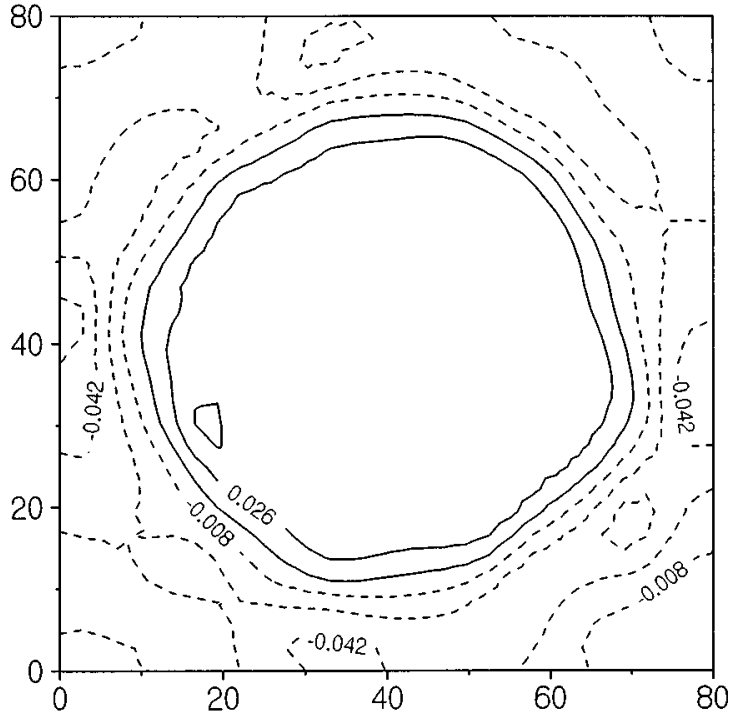

(a)

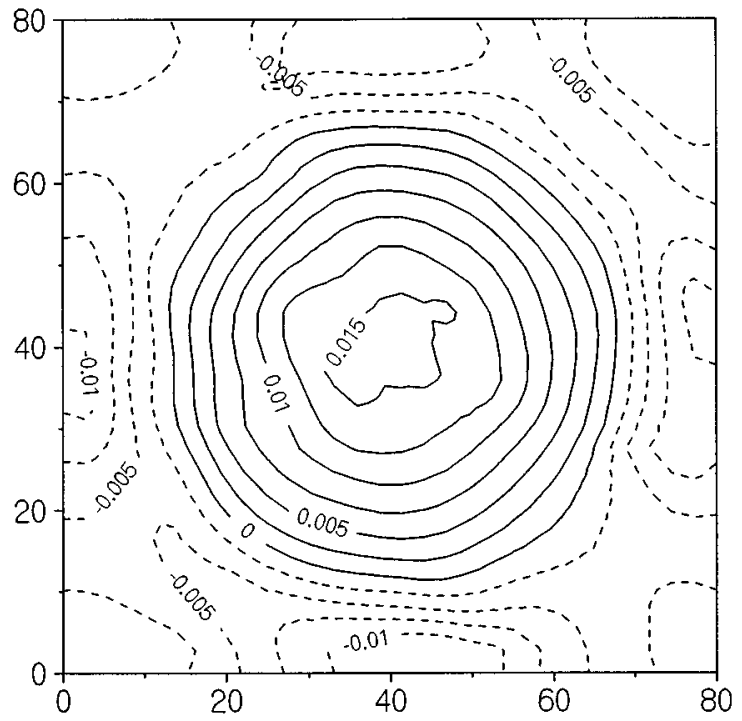

(C)

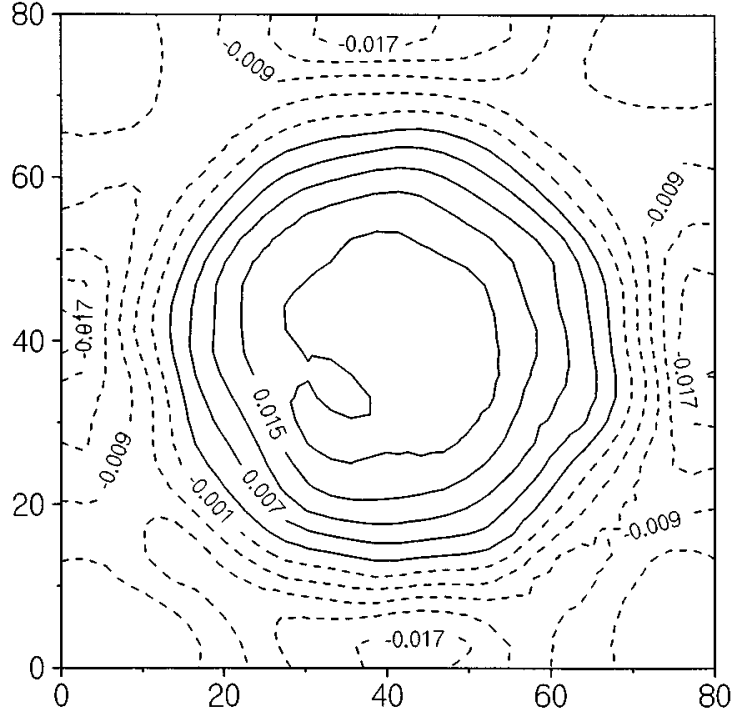

(b)

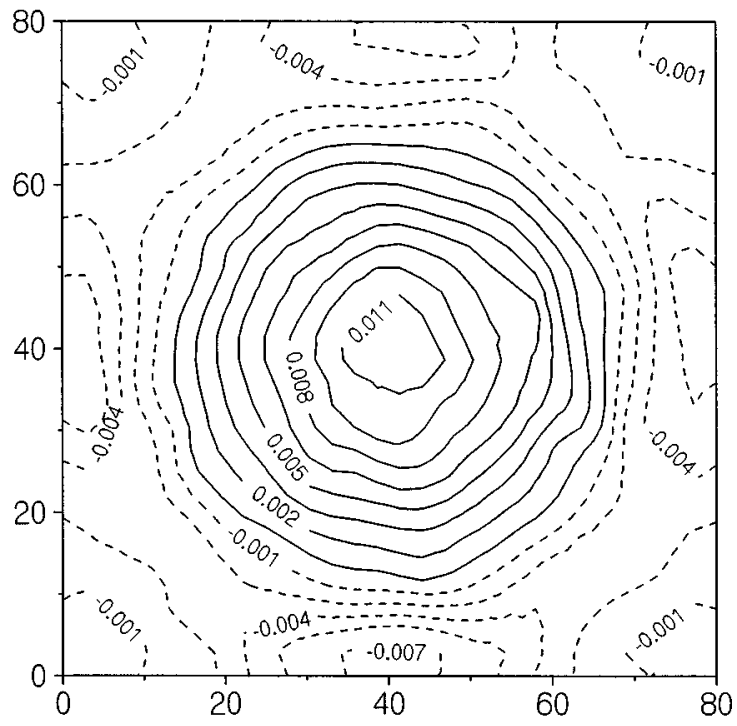

(d)

FIG. 6. Vorticity-contour plots of the jet-forced flow at (a) $35 \mathrm{~min}$; (b) $85 \mathrm{~min}$; (c) $115 \mathrm{~min}$ and (d) $145 \mathrm{~min}$. Experimental parameters: $Q=4 \mathrm{ml} / \mathrm{s}$, duration of forcing $10 \mathrm{~min}$.

$$
\begin{aligned}
\psi_{2}= & \sum_{n=0}^{\infty} B_{n} \frac{\cos \left(n+\frac{1}{2}\right) \pi y / a}{(-1)^{n}\left(n+\frac{1}{2}\right)} \\
& \times\left(\frac{\cosh \sqrt{\left(n+\frac{1}{2}\right)^{2}-\mu} \pi x / a}{\cosh \sqrt{\left(n+\frac{1}{2}\right)^{2}-\mu} \pi}-\frac{\cosh \left(n+\frac{1}{2}\right) \pi x / a}{\cosh \left(n+\frac{1}{2}\right) \pi}\right) .
\end{aligned}
$$

Both series satisfy the differential equation (10) and the condition that $\psi=0$ for $x= \pm a$ and for $y= \pm a$. In this notation, $\left(m+\frac{1}{2}\right)^{2}-\mu$ and $\left(n+\frac{1}{2}\right)^{2}-\mu$ are allowed to become negative; in that case the hyperbolic cosine should be interpreted as the cosine of a real argument. The combination of these two series provides the right number of parameters required to satisfy the remaining boundary conditions $\partial \psi / \partial x=0$ at $x= \pm a$ and $\partial \psi / \partial y=0$ at $y= \pm a$. Application of the boundary condition at $x=a$ yields

$$
\begin{aligned}
& -\sum_{m=0}^{\infty} A_{m}\left(\frac{\cosh \sqrt{\left(m+\frac{1}{2}\right)^{2}-\mu} \pi y / a}{\cosh \sqrt{\left(m+\frac{1}{2}\right)^{2}-\mu} \pi}-\frac{\cosh \left(m+\frac{1}{2}\right) \pi y / a}{\cosh \left(m+\frac{1}{2}\right) \pi}\right) \\
& +\sum_{n=0}^{\infty} B_{n} \frac{(-1)^{n}}{n+\frac{1}{2}}\left(\sqrt{\left(n+\frac{1}{2}\right)^{2}-\mu} \tanh \sqrt{\left(n+\frac{1}{2}\right)^{2}-\mu} \pi\right. \\
& \left.-\left(n+\frac{1}{2}\right) \tanh \left(n+\frac{1}{2}\right) \pi\right) \cos \left(n+\frac{1}{2}\right) \pi y / a=0 .
\end{aligned}
$$

Expanding the hyperbolic cosines in $y$ in $\cos \left(n+\frac{1}{2}\right) \pi y$ using 

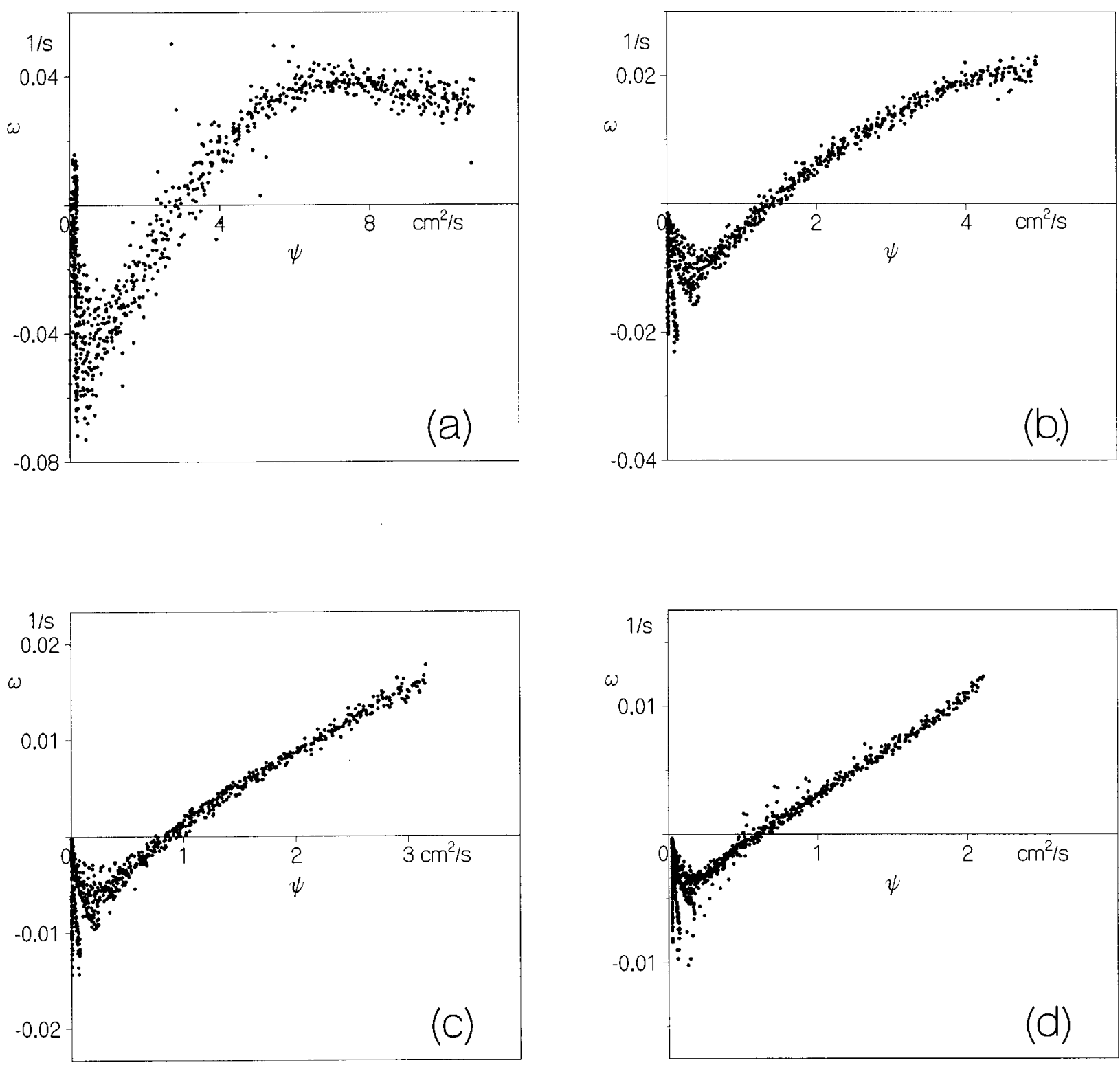

FIG. 7. Scatterplots of the jet-forced flow at (a) $35 \mathrm{~min}$; (b) $85 \mathrm{~min}$; (c) $115 \mathrm{~min}$ and (d) $145 \mathrm{~min}$.

$$
\frac{\cosh k \pi y / a}{\cosh k \pi}=\frac{2}{\pi} \sum_{n=0}^{\infty} \frac{(-1)^{n}\left(n+\frac{1}{2}\right)}{\left(n+\frac{1}{2}\right)^{2}+k^{2}} \cos \left(n+\frac{1}{2}\right) \pi y / a
$$

yields

$$
\begin{aligned}
& -\frac{2}{\pi} \sum_{m=0}^{\infty} A_{m} \sum_{n=0}^{\infty}\left(\frac{(-1)^{n}\left(n+\frac{1}{2}\right)}{\left(n+\frac{1}{2}\right)^{2}+\left(m+\frac{1}{2}\right)^{2}-\mu}\right. \\
& \left.\quad-\frac{(-1)^{n}\left(n+\frac{1}{2}\right)}{\left(n+\frac{1}{2}\right)^{2}+\left(m+\frac{1}{2}\right)^{2}}\right) \cos \left(n+\frac{1}{2}\right) \pi y / a \\
& \quad+\sum_{n=0}^{\infty} B_{n} \frac{(-1)^{n}}{n+\frac{1}{2}}\left(\sqrt{\left(n+\frac{1}{2}\right)^{2}-\mu} \tanh \sqrt{\left(n+\frac{1}{2}\right)^{2}-\mu} \pi\right. \\
& \left.-\left(n+\frac{1}{2}\right) \tanh \left(n+\frac{1}{2}\right) \pi\right) \cos \left(n+\frac{1}{2}\right) \pi y / a=0 .
\end{aligned}
$$

Collecting the coefficients of $\cos \left(n+\frac{1}{2}\right) \pi y / a$ yields

$$
\begin{aligned}
& -\frac{2}{\pi} \sum_{m=0}^{\infty} A_{m}\left(\frac{\left(n+\frac{1}{2}\right)^{2}}{\left(n+\frac{1}{2}\right)^{2}+\left(m+\frac{1}{2}\right)^{2}-\mu}-\frac{\left(n+\frac{1}{2}\right)^{2}}{\left(n+\frac{1}{2}\right)^{2}+\left(m+\frac{1}{2}\right)^{2}}\right) \\
& \quad+B_{n}\left(\sqrt{\left(n+\frac{1}{2}\right)^{2}-\mu} \tanh \sqrt{\left(n+\frac{1}{2}\right)^{2}-\mu} \pi\right. \\
& \left.\quad-\left(n+\frac{1}{2}\right) \tanh \left(n+\frac{1}{2}\right) \pi\right)=0
\end{aligned}
$$

and similarly, due to the boundary condition at $y=a$,

$$
\begin{aligned}
& A_{m}( \sqrt{\left(m+\frac{1}{2}\right)^{2}-\mu} \tanh \sqrt{\left(m+\frac{1}{2}\right)^{2}-\mu} \pi-\left(m+\frac{1}{2}\right) \\
&\left.\times \tanh \left(m+\frac{1}{2}\right) \pi\right)-\frac{2}{\pi} \sum_{n=0}^{\infty} B_{n}\left(\frac{\left(m+\frac{1}{2}\right)^{2}}{\left(m+\frac{1}{2}\right)^{2}+\left(n+\frac{1}{2}\right)^{2}-\mu}\right. \\
&\left.-\frac{\left(m+\frac{1}{2}\right)^{2}}{\left(m+\frac{1}{2}\right)^{2}+\left(n+\frac{1}{2}\right)^{2}}\right)=0 .
\end{aligned}
$$


This is an infinite system of linear equations in $A_{m}$ and $B_{n}$, which was solved numerically by truncating both the series over $m$ and over $n$ after 12 terms. The 12 terms in the series over $m$ and $n$ lead to 24 linear equations with 24 unknowns. Since the solution for a single mode is undetermined by a constant factor, this system of equations is dependent. The determinant being zero provides an equation for $\mu$; the remaining 23 equations were used to compute the ratios between the $A_{m}$ and $B_{n}$. This approach led to virtually the same results as a similar calculation with only 6 terms in each of the series over $m$ and $n$, so that we may assume that the influence of the truncation is negligible.

Starting from even functions in both $x$ and $y$, solutions of two symmetry types appear: type I with $A_{m}=B_{m}$ and $\psi(-y, x)=\psi(x, y)$, and type II with $A_{m}=-B_{m}$ and $\psi(-y, x)=-\psi(x, y)$. Thus, solutions of type I have a higher symmetry, being invariant under rotations over $\pi / 2$, whereas the highest rotational symmetry of solutions of type II is a rotation over $\pi$.

The results for the five lowest modes are presented in Fig. 8. The data for the lowest mode $(\mu=1.326)$ show a nearly linear relationship between $\omega$ and $\psi$ in the center of the tank. Close to the walls, the isoline patterns of $\omega$ and $\psi$ differ more, and the scatter in the $\omega, \psi$-plot is correspondingly higher. For higher modes, the stream function and vorticity fields have more structure. Taylor calculated only the lowest eigenvalue; his result agrees with the value of 1.326 in Fig. 8.

The eigenvalues can be compared with the eigenvalues of the Stokes flow on a circular domain. In that case, the vorticity and stream function corresponding to the azimuthally symmetric solutions are given by

$$
\begin{aligned}
& \omega(r, t)=\sum_{n=1}^{\infty} c_{n}\left(\frac{j_{1 n}^{2}}{a^{2}}\right) J_{0}\left(j_{1 n} r / a\right) \exp \left(-j_{1 n}^{2} \nu t / a^{2}\right), \\
& \psi(r, t)=\sum_{n=1}^{\infty} c_{n}\left[J_{0}\left(j_{1 n} r / a\right)-J_{0}\left(j_{1 n}\right)\right] \exp \left(-j_{1 n}^{2} \nu t / a^{2}\right)
\end{aligned}
$$

with $J_{0}$ the Bessel function of zero order, and $j_{1 n}$ the zeroes of the Bessel function of order one. Assuming that the time scale of the lowest mode in the square tank is approximately equal to that in a circular tank with the same area, we find that

$$
\mu_{n}=\frac{j_{1 n}^{2}}{4 \pi},
$$

which yields $\mu_{1}=1.168$ and $\mu_{2}=3.917$ for the two lowest modes. These values are about $10 \%$ lower than the exact results for a square tank, which is quite plausible, especially in view of the expectation that a more irregular domain will lead to a faster decay.

If we assume that the decaying flow is invariant under rotations over $\pi / 2$, the time scale on which the $\omega, \psi$-relation becomes linear is determined by the mode with $\mu=4.231$, since this is the first higher mode with the right symmetry type. For the tank of $80 \times 80 \mathrm{~cm}$ used in the experiments, this corresponds to a time scale of about $3800 \mathrm{~s}$.

\section{DISCUSSION AND CONCLUSIONS}

Both the experimental and numerical results indicate that independent of the type of initial forcing, the decaying twodimensional flow on a square domain tends to become organized in a single vortex. In the case of a flow that is forced for a finite time only, the graph of $\omega$ versus $\psi$ shows a slightly upward curvature, in qualitative agreement with the results of Montgomery and Joyce and Robert and Sommeria. In the case of a flow that is driven continuously by jets along the sidewalls, vorticity gradients close to the center disappear, and in spite of the square geometry the flow far from the sidewalls rotates like a rigid body. As the forcing is no longer applied, the flow gradually obtains an almost linear relationship between vorticity and stream function. Similar results were also found by Van Geffen, Meleshko, and Van Heijst, ${ }^{19}$ who studied the evolution of a decaying monopolar vortex on a square domain. Although the Reynolds number in both the experiments and the simulations is initially high, the ultimate flow pattern closely resembles the fundamental mode of a decaying viscous flow in the limiting case $R e=0$. This conclusion may be surprising at first sight, since the viscosity in flows with high Reynolds number is by definition very small. However, a smaller viscosity merely extends the diffusive time scale. On this longer time scale, there seems to be no escape for higher modes to be damped out. It is true that the presence of the advection term leads to a different flow than in the case $R e=0$, but these differences imply a more intricate velocity field, so that the vorticity will be distributed even faster.

We would like to emphasize the fact that the time scale on which the lowest mode is attained is much shorter than the roughest possible estimate, $L^{2} / \nu$. According to the results for the lowest mode but one (see Fig. 8), we arrive at a time scale $L^{2} /\left(4 \nu \pi^{2} 4.231\right)$, which is about 167 times shorter than $L^{2} / \nu$. Without taking notice of this large factor, one may seriously underestimate the influence of viscous diffusion.

The situation in the experiments is more complex than a purely two-dimensional flow; the motion in different layers of the stratified environment may be different, giving rise to viscous diffusion in the vertical direction and possibly internal Ekman-suction effects. The coupling between stratification and horizontal motion was discussed for an unbounded domain by Pearson and Linden. ${ }^{20}$ According to these authors, the interaction is strongest for small wavelengths, and they study in detail the coupling mechanism for $N \cos \theta \ll(\nu-\kappa) k^{2}$, with $k$ the wave number, $N$ the buoyancy frequency, $\theta$ the angle of the wavevector with the horizontal, and $\kappa$ the diffusivity of, for our case, salt. In the late stages of our experiments, we are in the opposite regime $N \cos \theta \gg(\nu-\kappa) k^{2}$, in which density-driven oscillations and decaying horizontal modes are decoupled. Still, large-scale oscillations may enhance the entrainment of vorticity at the sidewalls, and thereby influence the decay of the horizontal motion (see also Staquet and Riley ${ }^{21}$ for a numerical study on wave-vortex interaction). However, the dye visualizations in later stages show no evidence of wavelike motions, so this effect must be very small.

For horizontal motion $\left(\theta=\frac{1}{2} \pi\right)$ the theory of Pearson 


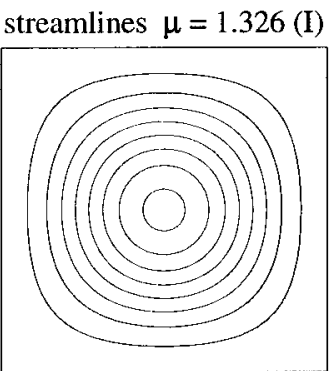

streamlines $\mu=3.904$ (II)

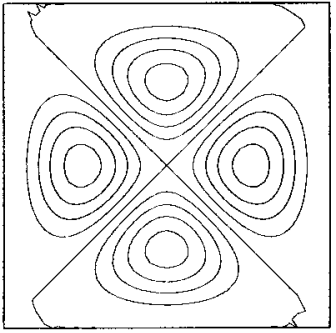

streamlines $\mu=4.231$ (I)

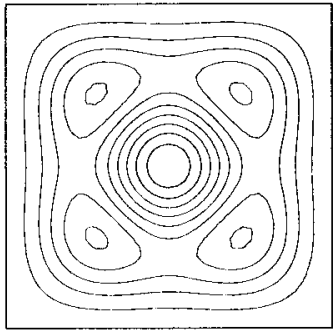

streamlines $\mu=6.240$ (I)

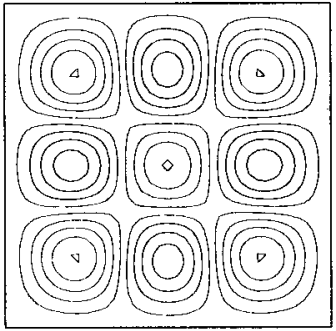

streamlines $\mu=8.848$ (II)

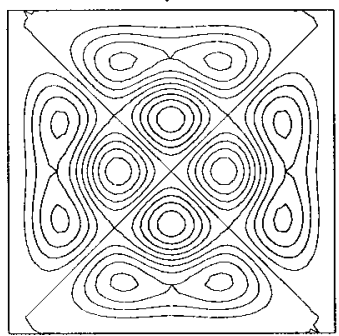

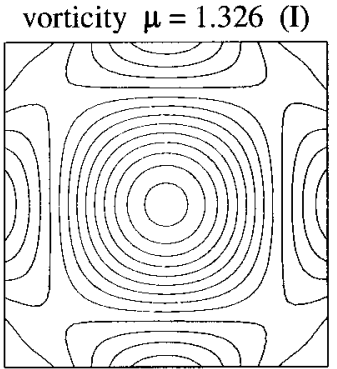
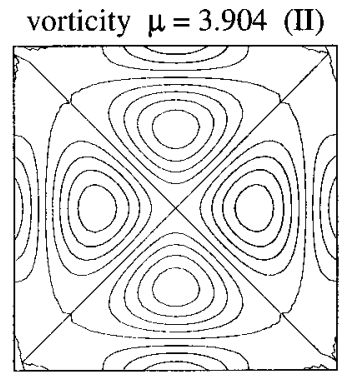

vorticity $\mu=4.231$ (I)
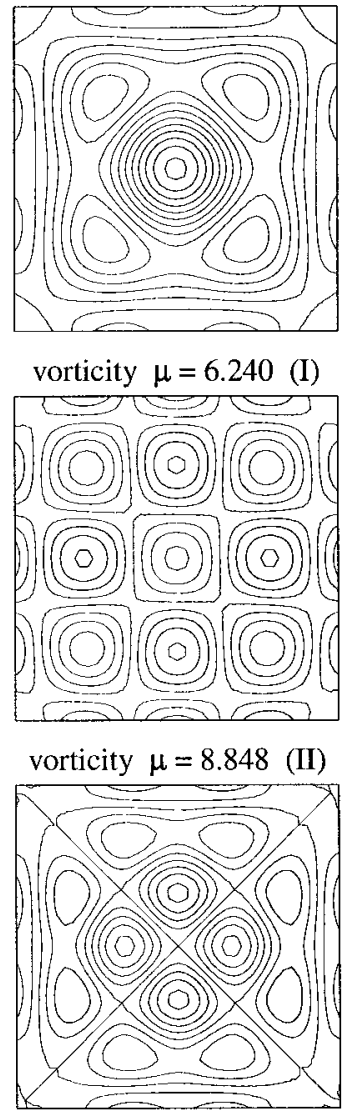
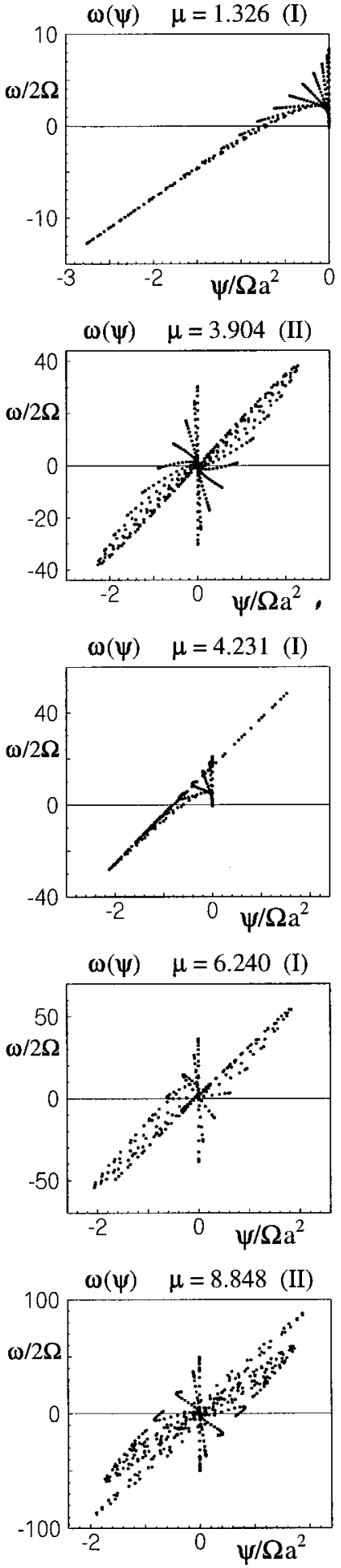

FIG. 8. The five lowest modes consisting of even functions in both the $x$ - and $y$-direction, obtained by a numerical solution of the linear set of $2 \times 12$ equations following from (18) and (19). Modes of type I are invariant under a rotation over $\pi / 2$, whereas modes of type II change sign under a rotation over $\pi / 2$. The fans of branches in the scatterplots have no physical meaning, but are caused by the corresponding points in the $x, y$-plane lying on a Cartesian grid.

and Linden cannot be applied. Nonetheless, in that case there may still be smoothing of the velocity profiles over different horizontal layers in the tank. For this reason, we expect viscous diffusion within a horizontal plane to provide an upper limit for the experimental decay time. In the experiments, the decay time is not significantly smaller than $3800 \mathrm{~s}$ (the value predicted by the decay analysis described in section V), indicating that after the forcing has stopped, three-dimensional effects are not dominant.

Both in the experiments and in the numerical simulations described in this study, the flow in the final stage was observed to consist of a single cell that fills the domain com- 
pletely, irrespective of the type of initial forcing. Apparently, the flow in this stage contains non-zero net angular momentum. This observation agrees with results obtained by highresolution spectral flow simulations on a square domain with no-slip boundaries by Clercx et al. ${ }^{16}$ In their simulations it was found that the net angular momentum of the flow generally increased (in absolute sense) in the first stage of the flow evolution, upon which it decayed very slowly compared with e.g. the total kinetic energy and the total enstrophy of the flow. This counter-intuitive behavior can be explained by the net torque produced by shear stress and normal stress (both viscous and pressure) acting at the walls of the square domain. Even in the non-realistic case of stress-free or freeslip walls, the flow in general acquires a non-zero angular momentum, merely due to the action of the wall pressure force that yields a net torque on the fluid. This behavior is in marked contrast with results obtained by Li, Montgomery and Jones ${ }^{22}$ for the evolution of a randomly initialized twodimensional flow on a circular domain with stress-free, freeslip or no-slip boundaries: in their simulations the flow in the final stage took on the appearance of a dipolar structure. The crucial difference between this particular geometry and the square domain is that the normal stress (either viscous or pressure) does not produce a net torque with respect to the center of the circular domain. Due to the critical dependence on the initial conditions, such differences are difficult to investigate experimentally. Experiments similar to those described in this paper have also been carried out in a circular domain by Flór, ${ }^{9}$ and lead to similar results as in a square tank. In particular, the flow is observed to become unidirectional, also in the case of a rake-forced initial disturbance. However, in experiments it is difficult to ensure that no initial angular momentum is imparted to the fluid, so that an agreement with the numerical results of Li et al. in this respect is not to be expected.

\section{ACKNOWLEDGMENTS}

The authors would like to thank Professor V. V. Meleshko, who recognized the similarity between the viscous flow on a rectangular domain and the bending of clamped plates, J. H. Voskamp for providing the numerical code, and F. Engels for his help by digitizing the particle streaks. Two of us (J.A.vdK. and J.B.F.) gratefully acknowledge financial support from the Dutch Foundation of Fundamental Research (FOM). Further, we want to thank Ris $\phi$ National Laboratory for giving J.A.vdK. the opportunity to finish the paper.
${ }^{1}$ J. C. McWilliams, "The emergence of isolated coherent vortices in turbulent flows," J. Fluid Mech. 146, 21 (1984).

${ }^{2}$ B. Legras, P. Santangelo, and R. Benzi, "High-resolution numerical experiments for forced two-dimensional turbulence," Europhys. Lett. 3, 811 (1988).

${ }^{3}$ B. M. Boubnov, S. B. Dalziel, and P. F. Linden, "Source-sink turbulence in a stratified fluid," J. Fluid Mech. 261, 273 (1994)

${ }^{4}$ D. Montgomery and G. Joyce, "Statistical mechanics of negative temperature states," Phys. Fluids 17, 1139 (1974).

${ }^{5}$ R. Robert and J. Sommeria, "Statistical equilibrium states for twodimensional flows," J. Fluid Mech. 229, 291 (1991).

${ }^{6}$ R. Salmon, G. Holloway, and M. C. Hendershott, "The equilibrium statistical mechanics of simple, quasi-geostrophic models," J. Fluid Mech. 75, 691 (1976).

${ }^{7}$ R. Robert and J. Sommeria, "Relaxation towards a statistical equilibrium state in two-dimensional perfect fluid dynamics," Phys. Rev. Lett. 69, 2776 (1992).

${ }^{8}$ D. Montgomery, X. Shan, and W. H. Matthaeus, "Navier-Stokes relaxation to sinh-Poisson states at finite Reynolds numbers,' Phys. Fluids A 5, 2207 (1993).

${ }^{9}$ J. B. Flór, Coherent Vortex Structures in Stratified Fluids, Ph.D. thesis, Eindhoven University of Technology, The Netherlands, 1994.

${ }^{10}$ J. B. Flór and G. J. F. van Heijst, " Experiments on dipolar structures in a stratified fluid," J. Fluid Mech. 279, 101 (1994).

${ }^{11}$ G. I. Taylor, "The buckling load for a rectangular plate with four clamped edges," Zeitschrift für angewandte Mathematik und Mechanik XIII, 147 (1993), reprinted in The Scientific Papers of Sir Geoffrey Ingram Taylor, Vol. I (Cambridge University Press, Cambridge, 1958).

${ }^{12}$ J. A. van de Konijnenberg, Spin-up in non-axisymmetric containers, Ph.D. thesis, Eindhoven University of Technology, The Netherlands, 1995.

${ }^{13}$ J. M. Nguyen Duc and J. Sommeria, "Experimental characterization of steady two-dimensional vortex couples," J. Fluid Mech. 192, 175 (1988).

${ }^{14}$ C. A. J. Fletcher, Computational Techniques for Fluid Dynamics 1: Fundamental Techniques and Computational Techniques for Fluid Dynamics 2: Specific Techniques for Different Flow Categories (Springer, Berlin, 1988).

${ }^{15}$ J. B. Flór and G. J. F. van Heijst, "Stable and unstable monopolar vortices in a stratified fluid," J. Fluid Mech. 311, 257 (1996).

${ }^{16}$ H. J. H. Clercx, S. R. Maassen, and G. J. F. van Heijst, “Decaying twodimensional turbulence in square containers with no-slip or stress-free boundaries," submitted to Phys. Fluids.

${ }^{17}$ S. P. Timoshenko, and S. Woinowsky-Krieger, Theory of Plates and Shells (McGraw-Hill, New York, 1970).

${ }^{18} \mathrm{~V}$. V. Meleshko and A. M. Gomilko, "On the bending of clamped rectangular plates,"' Mech. Res. Comm. 21, 19 (1994).

${ }^{19}$ J. H. G. M. van Geffen, V. V. Meleshko, and G. J. F. van Heijst, "Motion of a two-dimensional monopolar vortex in a bounded rectangular domain," Phys. Fluids 8, 2393 (1996).

${ }^{20} \mathrm{H}$. J. Pearson and P. F. Linden, "The final stage of decay of turbulence in stably stratified fluid,' J. Fluid Mech. 134, 195 (1983).

${ }^{21} \mathrm{~S}$. Li, D. Montgomery, and W. B. Jones, "Two-dimensional turbulence with rigid circular walls," Theor. Comput. Fluid Dyn., in press, 1997.

${ }^{22} \mathrm{C}$. Staquet and J. J. Riley, " On the velocity field associated with potential vorticity,’ Dyn. Atmos. Oceans 14, 93 (1989). 